\title{
Coordination Chemistry Reviews
}

\section{Highlights}

\section{Coordination chemistry and metal-involving reactions of amidoximes:} Relevance to the chemistry of oximes and oxime ligands

Dmitrii S. Bolotin, Nadezhda A. Bokach, Vadim Yu. Kukushkin*

- Synthesis, properties, and identification of metal-free amidoximes.

- Synthesis and structural features of amidoxime complexes.

- Comparison of chemistry of amidoximes with that of conventional oximes.

- Metal-mediated and metal-catalyzed reactions involving uncomplexed amidoximes.

- Reactions of amidoxime ligands. 